\title{
Cohort studies and the development of psychopathology: commentary on the Great Smoky Mountain Study
}

\author{
Matt McGue ${ }^{1}$
}

Received: 29 April 2016/Accepted: 11 May 2016/Published online: 23 May 2016

(C) Springer-Verlag Berlin Heidelberg 2016

In 1984, the developmental psychologist Dante Cicchetti [1] proclaimed the emergence of a new paradigm for the study of the development of mental illness, an approach he called developmental psychopathology. In contrast to the dominant research paradigms of that time that focused primarily on cross-sectional, case-control comparisons, developmental psychopathology emphasized the continuity between normal and abnormal development, the need for longitudinal methods to investigate the early manifestations of mental illness, and the importance of multidimensional, biopsychosocial approaches. Although warmly embraced, Cicchetti's pronouncement was more aspirational than descriptive. At the time, there was very little data to support the utility of the developmental psychopathology approach he was advocating.

That all began to change with the publication of findings from a series of landmark cohort studies, all initiated shortly before or just after the Cicchetti publication. These included the Avon Longitudinal Study of Parents and Children in the United Kingdom, the Dunedin and Christchurch birth cohort studies from New Zealand, the Montreal Longitudinal and Experimental Study in Canada, and the Great Smoky Mountains Study (GSMS) in the U.S. Although each of these studies was instrumental in providing an empirical foundation for the fledgling field of developmental psychopathology, the GSMS is unique in its focus on the prevalence and outcomes associated with

This comment refers to the article available at doi:10.1007/s00127015-1168-1.

Matt McGue

mcgue001@umn.edu

1 Department of Psychology/Elliott Hall, University of Minnesota, Minneapolis, MN 55455, USA childhood psychiatric disorders in a rural setting. As the overview article by Costello, Copeland and Angold (CCA) [2] published in the May 2016 issue effectively highlights, the GSMS has made important methodological contributions to longitudinal psychopathology research, documented the generalizability of observations made in urban settings, and helped to characterize the nature and structure of childhood psychopathology.

CCA frame the contributions of the GSMS around a distinction that will be familiar to most epidemiologists. Descriptive epidemiology seeks to characterize the distribution of disease, across persons, time and place. At a descriptive level, the GSMS has had an enormous impact on the field. It has shown that childhood psychiatric disorders: (1) carry as heavy burden in rural settings as they had previously been shown to carry in urban settings [3]; (2) are organized around two major dimensions of psychopathology, now recognized by the field as externalizing and internalizing [3]; and (3) exhibit substantial developmental continuity, although the precise nature of that continuity varies by disorder and gender [4].

Analytical epidemiology seeks to characterize the causal origins of disease. Although in practice the distinction between descriptive and analytical epidemiology is not always sharp, it is of interest that the three examples that CCA use to highlight the contributions of the GSMS - the role of puberty in depression, the influence of family income on psychological and social functioning, and the impact of childhood psychiatric problems on adult functioning - all fall squarely within the analytical domain. That CCA would focus on the analytical contributions of the GSMS is understandable; after all, a major justification for taking on the burden of a long-term cohort study is that it will ultimately provide unique insights into the developmental origins of disease. Nonetheless, while the 
advantages of longitudinal research are considerable, the method is not without limitations, especially when the goal is to draw the causal inferences most valued in analytical epidemiology.

Arguably the two greatest challenges to draw causal inferences in observational epidemiology involve the temporal sequencing of exposure and outcome (i.e., to rule out reverse causation), and the control for unmeasured confounders [5]. GSMS research linking puberty to depression illustrates the importance of carefully timed assessments in establishing the proper sequencing of exposure and outcome. Prior to the GSMS, the use of wide assessment intervals supported no more than the conclusion that a gender difference in depression prevalence emerged sometime during adolescence. In the GSMS, however, annual assessment of pubertal status throughout early and middle adolescence helped to establish that the prevalence of depression increased abruptly during midpuberty among girls but remained relatively unchanged among boys [6]. The linking of the emergence of the gender difference in depression to mid-puberty is highly significant in that it implicates a range of biological and social factors tied to pubertal change as potentially accounting for the gender difference in depression. Nonetheless, it does not unambiguously identify what the exact causes of the gender gap in depression are, as the problem of unmeasured confounders is as salient in a longitudinal study as it is with other observational research designs.

Epidemiologists have made use of various design options in an attempt to strengthen causal inference in observational research [7]. Although not a feature of the original GSMS design, during the course of the study a fortuitous event occurred, what CCA term a "natural experiment', which allowed the researchers to explore the impact of family poverty on childhood psychiatric symptoms. The GSMS included a sizable subsample of American Indians and an Indian casino opened during the course of the study and distributed profits to tribal members, lifting many Indian families that had been below the federal poverty level at the start of the study out of poverty. Children in families moving out of poverty showed a reduction in symptoms of behavioral disorders after the opening of the casino, while a similar reduction in symptoms was not observed among children in families that were either stably poor or stably non-poor [8].

The opening of the casino allowed GSMS researchers to establish a tight link between family poverty and childhood behavioral problems. But had they unequivocally established causality? Perhaps, yet a natural experiment is not a true randomized experiment and the exposure to the economic benefits from the casino may not have been ignorable. In the case of the casino opening, money was distributed to all Indian families, and thus likely ignorable at that level. Nonetheless, whether the cash benefits allowed the family to move out of poverty may have been related to characteristics of the family, and thus potentially non-ignorable. Thus, while the opening of a casino allowed the GSMS to provide stronger evidence in support of a causal effect of family income on child behavior than most studies that had preceded it; we cannot argue that by themselves these findings are definitive. As CCA note, strong inference still requires a convergence of evidence across multiple sources.

The power of the GSMS design benefitted greatly from an event totally outside the control of the investigators, the opening of a casino. Epidemiologists have similarly taken advantage of other chance events, such as hurricanes or the trauma of 9/11 [9], to strengthen inferences in observational research. Yet it would not be prudent to allow strong inference to depend entirely on fortuitous happenings. Fortunately, there are a growing number of tools to strengthen causal inference that is available to the epidemiologist, including sibling/twin comparisons, instrumental variable analysis and Mendelian randomization [7]. The current generation of cohort studies is building on the legacy of studies like the GSMS by taking advantage of these tools.

The Great Smoky Mountains Study is one of the landmark studies in developmental psychology. It helped establish the legitimacy of a new approach to the study of the origins of mental illness and has revealed much about the structure of mental health problems and their continuity across the lifespan. Successfully undertaking a cohort study of this scope and duration requires extraordinary effort and resourcefulness. The field is very fortunate that Jane Costello, Adrian Angold and their colleagues were willing to make this commitment.

\section{References}

1. Cicchetti D (1984) The emergence of developmental psychopathology. Child Dev 55(1):1-7

2. Costello EJ, Copeland W, Angold A (2016) The Great Smoky Mountain Study: developmental epidemiology in the southeastern United States. Soc Psychiatry Psychiatr Epidemiol. (In press)

3. Costello EJ et al (1996) The Great Smoky Mountains Study of youth-goals, design, methods, and the prevalence of DSM-III-R disorders. Arch Gen Psychiatry 53(12):1129-1136

4. Costello EJ et al (2003) Prevalence and development of psychiatric disorders in childhood and adolescence. Arch Gen Psychiatry 60(8):837-844

5. Foster EM (2010) Causal inference and developmental psychology. Dev Psychol 46(6):1454-1480 
6. Angold A, Costello EJ, Worthman CM (1998) Puberty and depression: the roles of age, pubertal status and pubertal timing. Psychol Med 28(1):51-61

7. Richmond RC et al (2014) Approaches for drawing causal inferences from epidemiological birth cohorts: a review. Early Hum Dev 90(11):769-780
8. Costello EJ et al (2003) Relationships between poverty and psychopathology - a natural experiment. JAMA, J Am Med Assoc 290(15):2023-2029

9. Kessler RC et al (2008) Trends in mental illness and suicidality after Hurricane Katrina. Mol Psychiatry 13(4):374-384 